\title{
Ischemic Colitis: Clinical Presentation, Localization in Relation to Risk Factors, and Long-Term Results
}

\author{
Philippe M. Glauser • Petra Wermuth • \\ Gieri Cathomas • Evelyn Kuhnt • Samuel A. Käser • \\ Christoph A. Maurer
}

Published online: 1 September 2011

(C) Société Internationale de Chirurgie 2011

\begin{abstract}
Background Ischemic colitis is commonly thought to occur most often in the left hemicolon close to the splenic flexure owing to insufficient blood supply near Griffith's point. This study investigates the colorectal localization pattern, the risk factors, and the long-term outcome of histologically proven ischemic colitis.

Methods Between 1996 and 2004, a total of 49 patients with a median age of 69 years (range 26-94 years) with colonoscopically assessed and histologically proven ischemic colitis were identified on behalf of the pathology database. Long-term results of 43 patients were evaluated retrospectively after a median interval of 79 months (range 6-163 months).

Results In 27 patients (55\%) more than one location was affected. We found 98 affected locations in 49 patients. The distribution of ischemic colitis in our group shows no significantly preferred location. In an exploratory analysis, the cecum, ascending colon, and right flexure were affected significantly more often if intake of a nonsteroidal antiinflammatory drug (NSAID) is documented. There was no
\end{abstract}

Glauser Philippe and Wermuth Petra contributed equally for this study.

P. M. Glauser · P. Wermuth · S. A. Käser · C. A. Maurer ( ()

Department of Surgery, Hospital of Liestal

(affiliated with the University of Basel),

4410 Liestal, Switzerland

e-mail: christoph.maurer@ksli.ch

G. Cathomas

Institute of Pathology, Hospital of Liestal, Liestal, Switzerland

E. Kuhnt

Clinical Trial Center, Faculty of Medicine,

University of Leipzig, Leipzig, Germany association between the location of ischemic colitis and a history of smoking, peripheral artery occlusive disease, coronary heart disease, diabetes, or malignant tumor.

Conclusions Ischemic colitis seems not to have a predisposing site of occurrence in the colorectum, especially Griffith's point which was not afflicted significantly more often than other sites. Frequently, ischemic colitis afflicts more than one colonic location. In patients being treated with NSAIDs, ischemic colitis was observed significantly more often in the right hemicolon. Recurrence of ischemic colitis seems to be rare.

\section{Introduction}

Ischemic colitis (IC) is the most prevalent form of gastrointestinal ischemia and accounts for 1 in 2000 hospital admissions [1]. It seems to be the second or third most frequent cause of hematochezia [2,3], and its clinical picture manifests mostly as self-limiting ischemia involving the mucosa and submucosa and rarely as acute fulminant ischemia, which may progress to necrosis and death [4]. The incidence of IC is most likely underestimated because the mild form often is of transient nature and misdiagnosed when patients suffer from other diseases such as inflammatory bowel disease or infectious colitis [5-7]. The incidence in the literature according to searches of bibliographic databases in general populations ranged from 4.5 to 44 cases per 100,000 person-years [8].

Ischemia of the colon was first described after ligation of the inferior mesenteric artery during aortic surgery or colon resection $[9,10]$. IC is a noninfectious inflammation of the bowel caused by intestinal blood flow that is inadequate to serve the metabolic demands of the colon in a specific region [1]. The etiology of IC is multifactorial [7, 11, 12]. 
Small-vessel diseases such as atherosclerosis, vasculitis, and amyloidosis can cause IC, as can major cardiovascular surgery and systemic hypoperfusion due to all kinds of situations involving shock [1]. A number of drugs are known to cause IC as a result of their vasoconstrictive effect, including digoxin, diuretics, estrogen, and nonsteroidal antiinflammatory drugs (NSAIDs). Additionally, IC can be associated with systemic diseases (e.g., systemic lupus erythematosus, Wegener granulomatosis, amyloidosis) [13-23].

There are only a few reports with a large number of patients suffering from IC [6, 24]. It is widely thought that IC predominantly occurs from the left side of the transverse colon to the descending colon. This colonic region includes Griffith's point, the point of critical blood supply between the vascular areas of the superior mesenteric artery (SMA) and the inferior mesenteric artery (IMA). Analyses of arteriographic studies show that vascular anastomosis at Griffith's point is present in 48 to $100 \%$ of individuals [25-27].

Overall, the left part of the colon seems to be affected by IC in $>75 \%$ of cases $[7,28]$. The right part of the colon including the ileocecal area can be affected as well, but the incidence is reported to be low [28, 29]. The incidence of IC in the rectum ranges from 4 to $16 \%$ [24]. Most studies are based on a small number of patients, however, and as our concerns about the correctness of the location dogma for IC were growing over the years we aimed to analyze the colorectal location pattern, the risk factors, and the longterm outcome of histologically proven IC.

\section{Patients and methods}

\section{Patients}

We performed a retrospective database query at the Institute of Pathology, Kantonsspital Liestal, in Liestal, Switzerland from 1996 to 2004 for patients with histologically confirmed IC. This department processes the histological examination for all county hospitals and most of the gastroenterologists in private practice in the district of Baselland. The analyzed tissue specimens were obtained either by endoscopy or surgery, with none by autopsy.

Inclusion criteria were a macroscopically segmental, circular inflammation of the colon without histological signs of inflammatory bowel disease (IBD). To differentiate from NSAID-induced colitis, patients with nummular inflammation, erosions, or ulceration were excluded. The histologic changes of IC include edema, distorted crypts, mucosal and submucosal hemorrhage, inflammatory infiltration, granulation tissue, intravascular platelet thrombi, and necrosis [30]. Patients with IBD usually have less crypt damage and more inflammation [31]. The presence of a hyalinized, hemorrhagic lamina propria and full-thickness mucosal necrosis may help differentiate pseudomembranous colonic ischemia from Clostridium difficile-induced colitis $[4,32]$.

The query initially identified 58 patients suffering from IC. Medical records of all 58 patients were analyzed for clinical, endoscopic, and pathologic data. Secondary diagnoses, such as peripheral artery occlusive disease (PAOD), coronary heart disease, diabetes, or hyperlipoproteinemia, and data about duration of illness, number of endoscopies, mode of therapy, body mass index, and tobacco use were collected from the medical records as well. NSAID intake was defined as daily intake of at least one NSAID dose over a time period of more than 2 weeks immediately before the symptoms occurred.

The location of ischemia was documented based on endoscopic or surgical findings. We used the following exclusion criteria: IBD present or in the history, infectious colonic inflammation, former abdominal surgery, and prior bowel obstruction. Two patients were excluded because of prior sigmoid volvulus, five patients tested positive for C. difficile, and two patients had acute diverticulitis, leaving a final number of 49 patients for further analyses.

In July 2007, follow-up was performed by questionnaire to obtain details about the long-term results. The median time of follow-up was 79 months (range 6-163 months). A complete follow-up was obtained from 43 of the 49 patients $(87.8 \%)$. Regarding the remaining patients, two patients died within 3 months after diagnosis of IC due to heart failure, and four patients did not provide concrete, reliable data.

Symptoms or colonoscopy were assessed as a secondary event if the patient was pain-free for at least 90 days following hospital discharge.

Statistical analysis

Categorical data are presented as absolute and relative frequencies and the continuous data as the median and range. Groups were tested for differences by Pearson's $\chi^{2}$ test, Fisher's exact test, or the Mann-Whitney $U$ test, as appropriate. Exact 95 percent confidence (95\% CI) limits for binomial proportion were computed by the $\mathrm{F}$ distribution method according to Collett. Statistical significance is defined as $P<0.05$. All analyses were performed by SAS 9.1.3 (SAS Institute, Cary, NC, USA).

The local ethics committee of Basel and Baselland (Ref. 139/10) approved the study.

\section{Results}

A total of 36 women and 13 men were included in the study (73.5 vs. $26.5 \%$, ratio $2.7: 1.0$ ). The median age at the time 
of diagnosis was 69 years (range 26-94 years). Female patients were significantly older: The median age was 71 years (range 27-94 years) for women versus 60 years for men (range 26-92 years), $P=0.036$. Details are summarized in Fig. 1.

\section{Location of disease}

More than one segment was affected in 27 patients $(55.1 \%)$. Overall, we found 98 affected locations in 49 patients. Ischemic pancolitis was diagnosed in two patients $(4.1 \%)$. The allocation to the segments was as follows: cecum, $n=11$ (11.2\%); ascending colon, $n=15$ (15.3\%); right flexure, $n=6(6.1 \%)$; transverse colon, $n=8(8.2 \%)$; left flexure, $n=11(11.2 \%)$; descending colon, $n=27$ (27.5\%); sigmoid, $n=14$ (14.3\%); rectum, $n=6(6.1 \%)$.

No location was affected with a significantly higher incidence than any other (Fig. 2).

In patients with NSAID intake, the cecum (37.0 vs. 4.6\%, $P=0.013$ ), ascending colon (44.4 vs. $13.6 \%$, $P=0.029$ ), and right flexure (22.2 vs. $0.0 \%, P=0.027$ ) were significantly more affected than in patients with no documented NSAID intake. The subgroup analysis for smoking, peripheral artery occlusive disease, coronary heart disease, diabetes, and malignant tumor revealed no preferred allocation among the segments. The allocation of risk factors to the site of IC is shown in Table 1.

\section{Duration of illness and clinical manifestation}

Most of the cases ( $n=28,57.1 \%$ ) were acute events with admission to the hospital within $24 \mathrm{~h}$ after the first symptoms. A total of 37 patients (75.5\%) were looking for medical help from a physician within 3 days. Only two patients had abdominal pain for more than 1 month. Both patients were of advanced age (85 and 89 years), were under continuous NSAID intake due to other diseases, and had no history of coronary heart disease or diabetes. The most common symptoms were hematochezia (69.4\%), persistent diarrhea (49.0\%), abdominal pain (44.9\%), and pseudoobstruction $(26.5 \%)$. All of the other clinical manifestations were seen in $<5 \%$.

The federal pathology department processes both inpatient and outpatient histological specimens. All cases of IC were, however, detected in inpatients. The distribution of the duration of illness until hospital admission is illustrated in Fig. 3.

\section{Endoscopy}

Almost all patients had colonoscopy for diagnosis $(n=46$; $93.9 \%$ ), and IC was always detected at the initial

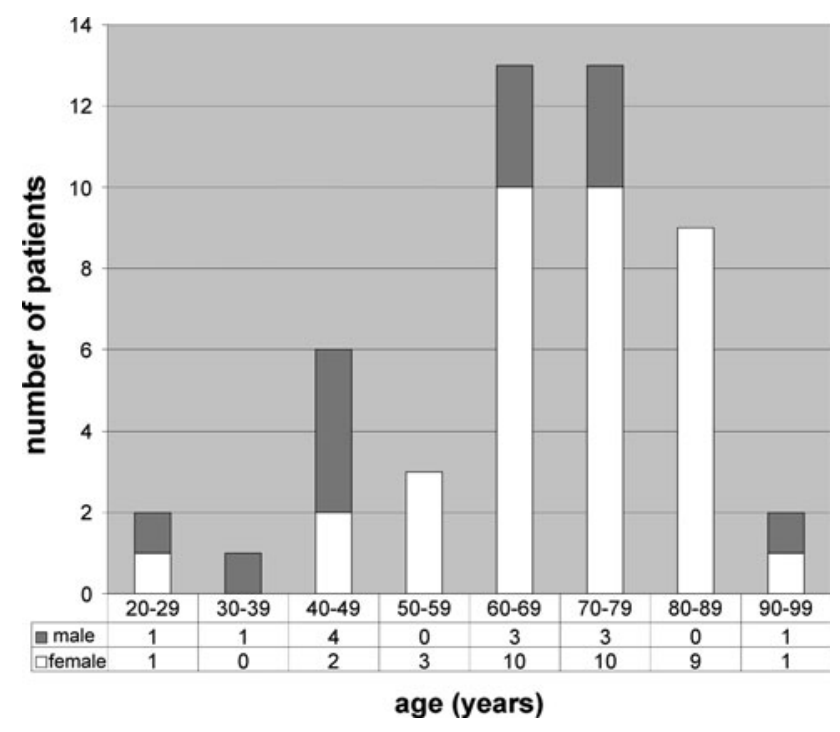

Fig. 1 Ischemic colitis (IC) with regard to age and sex
Fig. 2 Relative frequencies and 95\% confidence intervals of various locations with ischemic colitis

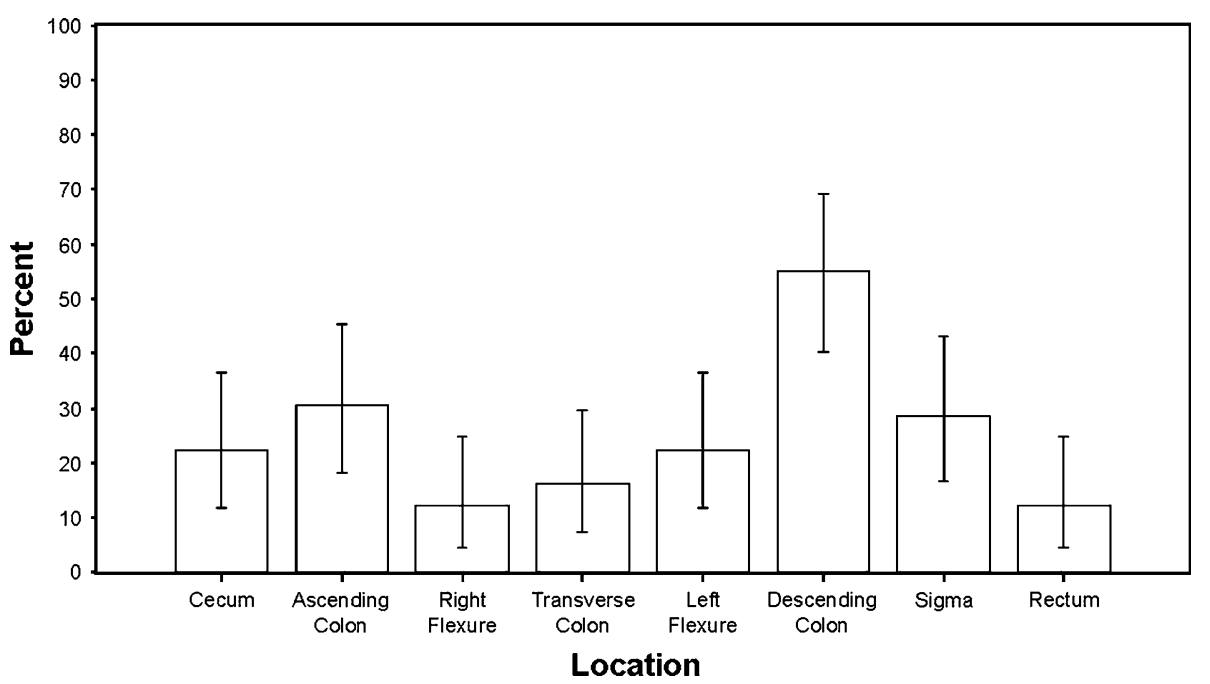


Table 1 Risk factors and sites of IC

\begin{tabular}{|c|c|c|c|c|c|c|c|c|c|}
\hline Factor & No. & $\begin{array}{l}\text { Cecum } \\
(n=11)\end{array}$ & $\begin{array}{l}\text { Ascending } \\
\text { colon } \\
(n=15)\end{array}$ & $\begin{array}{l}\text { Right } \\
\text { flexure } \\
(n=6)\end{array}$ & $\begin{array}{l}\text { Transverse } \\
\text { colon } \\
(n=8)\end{array}$ & $\begin{array}{l}\text { Left } \\
\text { flexure } \\
(n=11)\end{array}$ & $\begin{array}{l}\text { Descending } \\
\text { colon } \\
(n=27)\end{array}$ & $\begin{array}{l}\text { Sigmoid } \\
\text { colon } \\
(n=14)\end{array}$ & $\begin{array}{l}\text { Rectum } \\
(n=6)\end{array}$ \\
\hline Smoking & 17 & $4(23.5 \%)$ & $5(29.4 \%)$ & $1(5.9 \%)$ & $1(5.9 \%)$ & $3(17.7 \%)$ & $7(41.2 \%)$ & $6(35.3 \%)$ & 0 \\
\hline NSAIDs & 27 & $10(37.0 \%)$ & $12(44.4 \%)$ & $6(22.2 \%)$ & $6(22.2 \%)$ & $5(18.5 \%)$ & $13(51.8 \%)$ & $9(33.3 \%)$ & $4(14.8 \%)$ \\
\hline PAOD & 7 & 0 & $2(28.6 \%)$ & $1(14.3 \%)$ & $1(14.3 \%)$ & $2(28.6 \%)$ & $3(42.9 \%)$ & $2(28.6 \%)$ & 0 \\
\hline CHD & 14 & $1(7.1 \%)$ & $2(14.3 \%)$ & $1(7.1 \%)$ & $2(14.3 \%)$ & $3(21.4 \%)$ & $7(50.0 \%)$ & $6(42.9 \%)$ & $1(7.1 \%)$ \\
\hline Diabetes & 9 & $2(22.2 \%)$ & $5(55.5 \%)$ & $2(22.2 \%)$ & $2(22.2 \%)$ & $1(11.1 \%)$ & $3(33.3 \%)$ & $3(33.3 \%)$ & $2(22.2 \%)$ \\
\hline Malignancy & 5 & $1(20.0 \%)$ & $2(40.0 \%)$ & $1(20.0 \%)$ & $1(20.0 \%)$ & 0 & $2(40.0 \%)$ & $2(40.0 \%)$ & $1(20.0 \%)$ \\
\hline
\end{tabular}

NSAIDs nonsteroidal antiinflammatory drugs, $P A O D$ peripheral artery occlusive disease, $C H D$ coronary heart disease

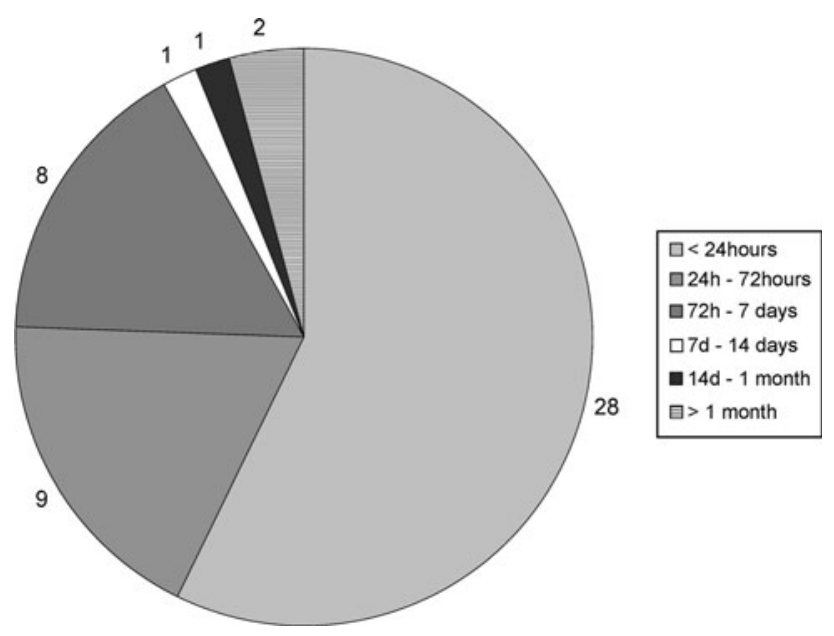

Fig. 3 Duration of symptoms of IC in 49 patients prior to hospital admission

colonoscopy. In all, 30 patients $(61.2 \%)$ had a single colonoscopy; 12 patients (24.5\%) were examined twice, 8 patients for the purpose of control and 4 patients had recurring symptoms; 3 patients $(6.1 \%)$ had three colonoscopies; and one patient underwent four examinations, all of which were for persistent symptoms. All colonoscopies were undertaken during the same hospital stay. In three patients $(6.1 \%)$, we diagnosed IC on a specimen resected by a surgeon.

\section{Therapy}

Most of the patients were treated conservatively $(n=45$; $91.8 \%$ ) with analgesics. Only four patients required surgery (8.2\%): Three underwent open right hemicolectomy and one sigmoid resection. One patient died after surgery because of multiorgan failure. None of the conservatively treated patients died within 90 days. The comparison of the mortality rates is not significant $(P=0.08)$.
Medication and secondary disease

More than half of the patients had a regular NSAID intake $(n=29,59.2 \%), 17$ patients $(34.7 \%)$ had a history of nicotine abuse (mean 35.8 pack years), and 14 patients (28.6\%) had a positive history of coronary heart disease. Only three patients had a positive family history of intestinal disease $(6.1 \%)$.

Blood lipid measurements were recorded in only 16 patients. Among them, 9 (18.4\%) patients had hyperlipoproteinemia.

In all, 29 patients $(59.2 \%)$ were overweight or with a higher weight according to the World Health Organization classification. Diabetes and malignant tumors were rare in our group, with only $9(18.4 \%)$ and $5(10.2 \%)$ cases, respectively.

None of the investigated precipitating causes for IC (smoking, peripheral occlusive arterial disease, coronary heart disease, diabetes, malignant tumor) were found in eight of the patients (16.3\%). Among the others, 17 patients $(34.7 \%)$ had a single cause and $24(49.0 \%)$ had multiple causes.

\section{Long-term results}

After a median observation time of 79 months (range 6-163 months), 36 of 43 patients (84\%) with reliable follow-up were completely free of further abdominal symptoms after the initial episode; 7 patients $(16 \%)$ had recurrent symptoms. The most frequent symptoms were abdominal pain, diarrhea, and mild hematochezia. Five of these patients underwent another colonoscopy (one patient twice), but in only two cases were signs of IC detectable. In one patient, IC affected the whole left hemicolon, whereas at the initial diagnosis of IC the disease was located solely in the sigmoid colon. The time interval was 4 years. In the other patient, IC was located in the splenic flexure (Griffith's point) only, whereas in the first episode IC was demonstrated in the whole left hemicolon. The time 
interval was 1 year. The results of the other three colonoscopies were one normal finding, one case of bacterial colitis, and one case of normal findings apart from hemorrhoids.

\section{Discussion}

Ischemic colitis is the most common form of intestinal ischemia. It occurs as the result of inadequate intestinal blood flow, which can result in a spectrum of injuries, from transient self-limited ischemia to transmural infarction.

Our study included 49 patients with histologically proven IC. The clinical manifestation of IC was observed more often in female than in male patients, as has also been described by Higgins et al. [8]. IC occurs at a significantly younger age in men than in women. The most frequent location was the descending colon followed by the ascending colon. In the literature, IC is mainly reported in the area from the left side of the transverse colon to the descending colon [7, 28, 33], which corresponds to the region of overlapping marginal blood supply due to the anastomosis between the superior and inferior mesenteric arteries [34], known as Griffith's point [26].

We did not observe a predisposition of this location for IC. In contrast, the allocation of IC to the various segments of colon is almost equally distributed between the left and right hemicolon. In 55\% (27/49) of the patients, two or more segments of the colon were afflicted by IC. Even in the rectum, which has a very good blood supply, IC was present in six patients (12.5\%) - as many as in the right flexure. Therefore, the authors assume that the pathophysiology of IC is caused by microembolism or vasoconstriction of the vasae rectae, which can affect the whole colorectum with no predominant location. Additionally, the finding of equal affection of the whole colorectum was also reported in recent studies. Brandt et al. [35] reported an affection of the left colon in only $32.6 \%$ of 313 patients. NSAID intake was not analyzed. Zou et al. found left hemicolon involvement in $80 \%$ of 85 patients, but only $14 \%$ affected the splenic flexure. The risk factors were reported but not analyzed for the various segments of the colon, and NSAID intake was not studied [36]. Acosta et al. studied 23,446 autopsies in which 53 cases of fatal IC were identified. They found splenic flexure involvement in $57 \%$, whereas the descending colon was affected in $77 \%$. This of course differs from our study in that only fatal cases of IC were considered [37]. Madoff et al. [38] also suggested that the watershed zone is not especially vulnerable and may even be considered ischemia-resistant based on the dual circulation in this region. With our data we showed only equal involvement of all segments of the colon.
In patients with NSAID intake, the locations of the right hemicolon were significantly more affected by IC. This is an interesting finding, particularly with regard to the fact that patients with signs of NSAID-induced colitis were excluded from this study. It may be related to the fact that drugs, which have not been reabsorbed completely in the small bowel, develop higher local drug concentrations in the right hemicolon than in the left hemicolon. In addition to the local toxic effects, which results in local inflammation, erosions, or ulceration, we assume that NSAIDs induce local vasoconstriction and promote IC.

None of the other evaluated risk factors showed a correlation with any colonic site in our study. Studies with a larger number of patients are necessary in future to give more information on this topic.

All cases of IC in our study were detected in inpatients, although the pathology department also processes outpatient specimens. It seems that the clinical manifestations of IC warrant immediate admission and colonoscopy, which can by provided only in a hospital. Of course, this finding depends mainly on the particular public health system.

To analyze the recurrence rate, we prospectively evaluated the long-term follow-up. Seven patients (16.3\%) had recurrent episodes of abdominal pain, diarrhea, or hematochezia; and five of these patients underwent re-colonoscopy. In only two patients $(4.6 \%)$ were control colonoscopy and biopsy able to demonstrate the persistence or recurrence of IC. In our study, IC was most often a transient, self-limiting disease that rarely required surgical intervention.

\section{Conclusions}

Our results indicated that every segment of the colon can be affected by IC. The area from the left part of the transverse colon to the descending colon (region of Griffith's point) did not show a significant higher incidence of IC than the right hemicolon. In more than half of the patients, IC had affected two or more segments of the colon. The right hemicolon was significantly more often affected than the left hemicolon in patients with NSAID intake. In most cases, IC is a mild, self-limiting disease that rarely requires surgical therapy. The recurrence rate of IC seems to be low. Brandt et al. [35] reported a significantly higher mortality rate for patients who required surgery. In our study, one patient died after surgery, whereas none died after conservative treatment, but the difference was not significant.

Conflicts of interest The authors have no conflicts of interest or financial ties to disclose. 


\section{References}

1. Elder K, Lashner BA, Al Solaiman F (2009) Clinical approach to colonic ischemia. Cleve Clin J Med 76:401-409

2. Longstreth GF (1997) Epidemiology and outcome of patients hospitalized with acute lower gastrointestinal hemorrhage: a population-based study. Am J Gastroenterol 92:419-424

3. Vernava AM 3rd, Moore BA, Longo WE et al (1997) Lower gastrointestinal bleeding. Dis Colon Rectum 40:846-858

4. Green BT, Tendler DA (2005) Ischemic colitis: a clinical review. South Med J 98:217-222

5. Theodoropoulou A, Koutroubakis IE (2008) Ischemic colitis: clinical practice in diagnosis and treatment. World J Gastroenterol 14:7302-7308

6. Scharff JR, Longo WE, Vartanian SM et al (2003) Ischemic colitis: spectrum of disease and outcome. Surgery 134:624-629; discussion 629-630

7. MacDonald PH (2002) Ischaemic colitis. Best Pract Res Clin Gastroenterol 16:51-61

8. Higgins PD, Davis KJ, Laine L (2004) Systematic review: the epidemiology of ischaemic colitis. Aliment Pharmacol Ther 19:729-738

9. Shaw RS, Green TH Jr (1953) Massive mesenteric infarction following inferior mesenteric-artery ligation in resection of the colon for carcinoma. N Engl J Med 248:890-891

10. Smith RF, Szilagyi DE (1960) Ischemia of the colon as a complication in the surgery of the abdominal aorta. Arch Surg 80:806-821

11. Izbicki JR, Schneider CG, Kastl S (2003) Partial ischemia: occlusive and nonocclusive mesenteric ischemia, ischemic colitis, systemic lupus erythematosus. Chirurg 74:413-418

12. Zelenock GB, Strodel WE, Knol JA et al (1989) A prospective study of clinically and endoscopically documented colonic ischemia in 100 patients undergoing aortic reconstructive surgery with aggressive colonic and direct pelvic revascularization, compared with historic controls. Surgery 106:771-779

13. Brown DN, Rosenholtz MJ, Marshall JB (1994) Ischemic colitis related to cocaine abuse. Am J Gastroenterol 89:1558-1561

14. Deana DG, Dean PJ (1995) Reversible ischemic colitis in young women: association with oral contraceptive use. Am J Surg Pathol 19:454-462

15. Patel YJ, Scherl ND, Elias S et al (1992) Ischemic colitis associated with psychotropic drugs. Dig Dis Sci 37:1148-1149

16. Johnson TD, Berenson MM (1991) Methamphetamine-induced ischemic colitis. J Clin Gastroenterol 13:687-689

17. Knudsen JF, Friedman B, Chen M et al (1998) Ischemic colitis and sumatriptan use. Arch Intern Med 158:1946-1948

18. Tada H, Saitoh S, Nakagawa Y et al (1996) Ischemic colitis during interferon-alpha treatment for chronic active hepatitis C. J Gastroenterol 31:582-584

19. Green BT, Branch MS (2003) Ischemic colitis in a young adult during sickle cell crisis: case report and review. Gastrointest Endosc 57:605-607
20. Kistin MG, Kaplan MM, Harrington JT (1978) Diffuse ischemic colitis associated with systemic lupus erythematosus: response to subtotal colectomy. Gastroenterology 75:1147-1151

21. Yee NS, Guerry DT, Lichtenstein GR (2000) Ischemic colitis associated with factor V Leiden mutation. Ann Intern Med 132:595-596

22. Richardson SC, Willis J, Wong RC (2003) Ischemic colitis, systemic lupus erythematosus, and the lupus anticoagulant: case report and review. Gastrointest Endosc 57:257-260

23. Byrd RL, Cunningham MW, Goldman LI (1987) Nonocclusive ischemic colitis secondary to hemorrhagic shock. Dis Colon Rectum 30:116-118

24. Longo WE, Ballantyne GH, Gusberg RJ (1992) Ischemic colitis: patterns and prognosis. Dis Colon Rectum 35:726-730

25. Binns JC, Isaacson P (1978) Age-related changes in the colonic blood supply: their relevance to ischaemic colitis. Gut 19:384-390

26. Meyers MA (1976) Griffiths' point: critical anastomosis at the splenic flexure. Significance in ischemia of the colon. AJR Am J Roentgenol 126:77-94

27. Steward JA, Rankin FW (1933) Blood supply of the large intestine: its surgical considerations. Arch Surg 26:843-891

28. Bower TC (1993) Ischemic colitis. Surg Clin North Am 73:1037-1053

29. Yamazaki T, Shirai Y, Tada T et al (1997) Ischemic colitis arising in watershed areas of the colonic blood supply: a report of two cases. Surg Today 27:460-462

30. Price AB (1990) Ischaemic colitis. Curr Top Pathol 81:229-246

31. Hunt RH, Buchanan JD (1979) Transient ischaemic colitis: colonoscopy and biopsy in diagnosis. J R Nav Med Serv 65:15-19

32. Dignan CR, Greenson JK (1997) Can ischemic colitis be differentiated from $C$. difficile colitis in biopsy specimens? Am J Surg Pathol 21:706-710

33. Gandhi SK, Hanson MM, Vernava AM et al (1996) Ischemic colitis. Dis Colon Rectum 39:88-100

34. Hunter GC, Guernsey JM (1988) Mesenteric ischemia. Med Clin North Am 72:1091-1115

35. Brandt LJ, Feuerstadt P, Blaszka MC (2010) Anatomic patterns, patient characteristics, and clinical outcomes in ischemic colitis: a study of 313 cases supported by histology. Am J Gastroenterol 105:2245-2252

36. Zou X, Cao J, Yao Y et al (2009) Endoscopic findings and clinicopathologic characteristics of ischemic colitis: a report of 85 cases. Dig Dis Sci 54:2009-2015

37. Acosta S, Ogren M, Sternby $\mathrm{NH}$ et al (2006) Fatal colonic ischemia: a population-based study. Scand J Gastroenterol 41:1312-1319

38. Carlson RM, Madoff RD (2011) Is "ischemic" colitis ischemic? Dis Colon Rectum 54:370-373 